\title{
On the first $\delta$ Scuti-like pulsating Ap star in an eclipsing binary
}

\author{
M. Skarka ${ }^{1,2}$, P. Kabáth ${ }^{2}$, E. Paunzen ${ }^{1}$, M. Mašek ${ }^{3}$, J. Žák ${ }^{1}$ and \\ J. Janík ${ }^{1}$ \\ 1 Department of Theoretical Physics and Astrophysics, Masaryk University, \\ Kotlářská 2, 61137 Brno, (E-mail: maska@physics.muni.cz) \\ 2 Astronomical Institute of the Czech Academy of Sciences, Fričova 298, \\ 25165 Ondřejov, The Czech Republic \\ 3 Institute of Physics, Czech Academy of Sciences, Na Slovance 1999/2, \\ CZ-18221 Praha, Czech Republic
}

Received: October 28, 2019; Accepted: December 3, 2019

\begin{abstract}
HD 99458 was recently discovered to be a first object of its kind: a short-period eclipsing binary hosts a spotted, chemically peculiar A-type primary star that is a $\delta$ Scuti pulsator, and an M-dwarf secondary. These phenomena should not co-exist at the same time. Based on new photometric data we find that the previously published period of the main pulsation mode is a Nyquist alias of the true period $(0.036 \mathrm{~d}$, and not $0.052 \mathrm{~d}$ ) given by the Kepler sampling.

Key words: Stars: HD 99458 - Stars: chemically peculiar - binaries: eclipsing - Stars: pulsations
\end{abstract}

\section{Introduction}

The class of magnetic chemically peculiar stars that show rotational variations due to spots (CP2 class) is characterized by an overabundance of heavy elements (Preston, 1974). From the interaction with the secondary component, a magnetic field is not expected in close binaries and only a few candidates are known (e.g. Carrier et al., 2002; Landstreet et al., 2017). Pulsations of $\delta$ Scuti type also have been rarely seen among CP2 stars (Bowman et al., 2018). Here we provide additional results on the reported discovery of a binary star with an A-type CP2 star that shows all the mentioned properties.

\section{HD 99458}

Skarka et al. (2019) discovered HD 99458 to be a 2.72-day eclipsing binary that shows rotational modulation, overabundance of $\mathrm{Si}, \mathrm{Ti}$ and other elements, and eclipses that were assumed to be due to a planetary transit (Barros et al., 2016). Skarka et al. (2019) showed that the companion is a $0.45 M_{\odot}$ M-dwarf star. 
Multi-mode stellar pulsations with the main period of $19.2 \mathrm{c} / \mathrm{d}$ were detected in the Kepler K2 data.

After obtaining new photometric data in 2019 (Fig. 1, left panel), we found that the main pulsation frequency originally detected in the $K 2$ data is actually the Nyquist alias of the true frequency (Fig. 1, right-hand panel).
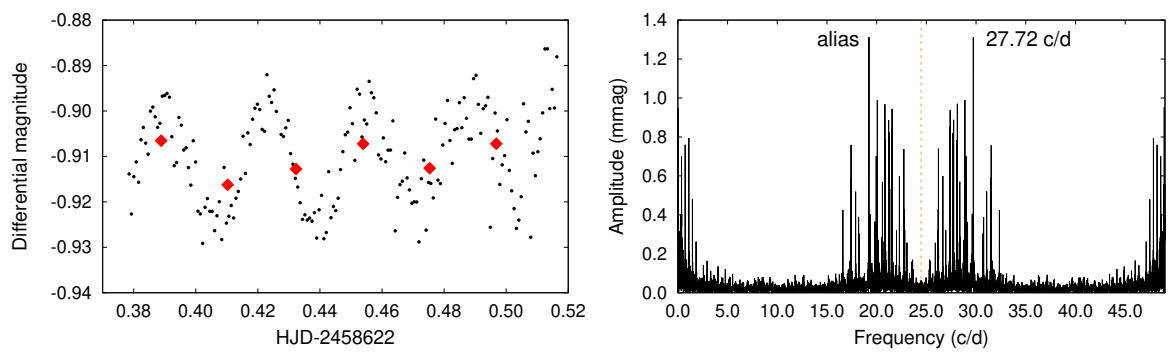

Figure 1. Data in Johnson $B$ (left panel) and frequency spectra from the Kepler K2 mission with identification of the main pulsation frequency and its alias. The red diamonds in the left panel are 30-min bins simulating the $K 2$ cadence.

Acknowledgements. MS acknowledges the OP VVV project Postdoc@MUNI No. CZ.02.2.69/0.0/0.0/16-027/0008360.

\section{References}

Barros, S. C. C., Demangeon, O., \& Deleuil, M., New planetary and eclipsing binary candidates from campaigns 1-6 of the K2 mission. 2016, Astron. Astrophys., 594, A100, DOI: 10.1051/0004-6361/201628902

Bowman, D. M., Buysschaert, B., Neiner, C., et al., K2 space photometry reveals rotational modulation and stellar pulsations in chemically peculiar A and B stars. 2018, Astron. Astrophys., 616, A77, DOI: 10.1051/0004-6361/201833037

Carrier, F., North, P., Udry, S., \& Babel, J., Multiplicity among chemically peculiar stars. II. Cool magnetic Ap stars. 2002, Astron. Astrophys., 394, 151, DOI: 10.1051/0004-6361:20021122

Landstreet, J. D., Kochukhov, O., Alecian, E., et al., BD-19 5044L: discovery of a short-period SB2 system with a magnetic Bp primary in the open cluster IC 4725. 2017, Astron. Astrophys., 601, A129, DOI: 10.1051/0004-6361/201630233

Preston, G. W., The chemically peculiar stars of the upper main sequence. 1974, Ann. Rev. Astron. Astrophys., 12, 257, DOI: 10.1146/annurev.aa.12.090174.001353

Skarka, M., Kabáth, P., Paunzen, E., et al., HD 99458: First time ever Ap-type star as a $\delta$ Scuti pulsator in a short period eclipsing binary? 2019, Mon. Not. R. Astron. Soc., 487, 4230, DOI: 10.1093/mnras/stz1478 